\title{
Avaliação da tenotomia ou tenodese bicipital na infiltração gordurosa do músculo bíceps*
}

\section{Evaluation of Biceps Tenotomy or Tenodesis on Fatty Infiltration of the Biceps Muscle}

\author{
Jair Simmer Filho ${ }^{10}$ Paulo Henrique Schmidt Lara ${ }^{2}$ \\ Benno Ejnisman ${ }^{2}$ \\ ${ }^{1}$ Departamento de Ortopedia e Traumatologia, Hospital Estadual de \\ Urgência e Emergência, Vitória, ES, Brasil \\ ${ }^{2}$ Centro de Traumatologia do Esporte, Escola Paulista de Medicina, \\ Universidade Federal de São Paulo, São Paulo, SP, Brasil \\ 3 Departamento de Radiologia, Multiscan Diagnóstico, Vitória, ES, Brasil \\ Rev Bras Ortop 2021;56(4):497-503.
}

Juarez Leite Júnior ${ }^{3}$ Paulo Santoro Belangero ${ }^{2}$ (잉

\begin{abstract}
Endereço para correspondência Paulo Henrique Schmidt Lara, MD, Rua Estado de Israel, 636, Vila Clementino, São Paulo, SP, 04022-001, Brasil (e-mail: phslara@gmail.com).
\end{abstract}

\section{Resumo}

Palavras-chave

- bíceps

- manguito rotador

- tenodese

- tenotomia
Objetivo O objetivo do presente estudo foi determinar a existência de infiltração gordurosa (IG) na massa muscular do bíceps braquial após a tenotomia ou tenodese para tratamento de lesão no tendão da cabeça longa do bíceps e estabelecer uma relação entre a IG e alterações no comprimento das fibras musculares.

Métodos Análise clínica e de imagens de 2 grupos de pacientes (submetidos à tenodese do bíceps [ 16 indivíduos] ou tenotomia do bíceps [15 indivíduos]). Nos dois grupos, os achados foram comparados àqueles do lado contralateral de cada indivíduo (grupo controle). Todos os pacientes foram submetidos à tenodese ou tenotomia unilateral do bíceps, com acompanhamento pós-operatório $>1$ ano. Exames de ressonância magnética (RM) foram realizados em ambos os braços de cada paciente de acordo com um protocolo específico. A força de flexão do cotovelo foi medida com dinamômetro manual e os resultados foram submetidos à análise estatística.

Resultados $\mathrm{O}$ período pós-operatório médio antes da realização da RM foi de 5 anos, e nenhum caso de IG foi observado no compartimento anterior de ambos os braços dos pacientes avaliados. Sete pacientes apresentaram deformidade moderada ou grave no braço operado. Não houve relação significativa entre deformidade do braço $(p=0,077)$, percentual de força de flexão $(p=0,07)$ ou dor à palpação do sulco bicipital $(p=0,103)$. Conclusão Nenhum dos pacientes avaliados apresentou evidência de IG na massa muscular do compartimento anterior do braço após os procedimentos. Não foi possível

Trabalho desenvolvido no Centro de Traumatologia do Esporte da Escola Paulista de Medicina, Universidade Federal de São Paulo, São Paulo, SP, Brasil.

recebido

01 de Dezembro de 2019

aceito

01 de Junho de 2020

Publicado on-line

Setembro 25, 2020
DOI https://doi.org/

$10.1055 / \mathrm{s}-0040-1714231$. ISSN 0102-3616. (c) 2020. Sociedade Brasileira de Ortopedia e Traumatologia. All rights reserved.

This is an open access article published by Thieme under the terms of the Creative Commons Attribution-NonDerivative-NonCommercial-License, permitting copying and reproduction so long as the original work is given appropriate credit. Contents may not be used for commercial purposes, or adapted, remixed, transformed or built upon. (https://creativecommons.org/ licenses/by-nc-nd/4.0/)

Thieme Revinter Publicações Ltda., Rua do Matoso 170, Rio de Janeiro, RJ, CEP 20270-135, Brazil 


\begin{abstract}
Keywords

- biceps

- rotator cuff

- tenodesis

- tenotomy

Objective The objective of the present study was to determine whether there is fatty infiltration (FI) of the biceps brachii muscle mass after tenotomy or tenodesis for the treatment of tendon injuries in the long head of the biceps and to establish a relationship between $\mathrm{Fl}$ with changes in the length of muscle fibers.

Methods Clinical and imaging analysis of 2 groups of patients (biceps tenodesis [16 patients] and biceps tenotomy [15 patients]). In both groups, we compared the findings on the contralateral side of each patient (control group). All patients had undergone unilateral biceps tenodesis or tenotomy, with postoperative follow-up of $>1$ year. Magnetic resonance imaging (MRI) was performed on both arms of each patient following a specific protocol. Strength of elbow flexion was measured with a manual dynamometer, and the results were subjected to statistical analysis.

Results The mean postoperative period before the MRI was 5 years, and no case of FI was observed in the anterior compartment of either arm of the evaluated patients. Seven patients had moderate or severe deformity in the operated arm. We found no significant relationship between arm deformity $(p=0.077)$, flexion strength percentage $(p=0.07)$ or pain on palpation of the bicipital groove $(p=0.103)$.

Conclusion None of the evaluated patients had evidence of $\mathrm{Fl}$ in the muscle mass of the anterior arm compartment after the procedures. It was not possible to establish a correlation between the discrepancy of the biceps muscle length measured by MRI and the presence of $\mathrm{Fl}$ in the anterior compartment of the arm.
\end{abstract}

estabelecer uma correlação entre a discrepância do comprimento do músculo bíceps, medido à RM, e a presença de IG no compartimento anterior do braço.

\section{Introdução}

Quando o tendão da cabeça longa do bíceps (TCLB) sofre ruptura ou é submetido a tenotomia ou tenodese, a retração do ventre muscular pode alterar a força e/ou a estética do braço. ${ }^{1}$ No entanto, a quantidade de retração capaz de provocar repercussões clínicas ainda não foi bem definida. Atrofia muscular, fibrose intersticial e infiltração gordurosa (IG) são frequentemente associadas a lesões crônicas do manguito rotador. Essas alterações estruturais irreversíveis favorecem a perda de força e elasticidade muscular e são grandes obstáculos ao sucesso do reparo cirúrgico de músculos como subescapular, supraespinhal e infraespinhal. ${ }^{2}$ Apesar de muito estudado, não está bem estabelecido se o encurtamento crônico da massa muscular do bíceps pode causar uma alteração no ventre muscular semelhante àquela provocada pela IG no ventre dos músculos do manguito rotador em casos de rupturas crônicas acompanhadas por retração. ${ }^{3,4}$

Em meados do século XX, oTCLB era visto como a principal fonte de dor no ombro, cujo tratamento era baseado em tenotomia e tenodese. ${ }^{5-7}$ Hoje, esses procedimentos para o tratamento de lesões relacionadas ao TCLB são amplamente realizados durante artroscopias do ombro. ${ }^{8,9}$ Não há um consenso claro sobre o melhor tratamento cirúrgico para lesões do TCLB ${ }^{10}$ Além disso, não existe um método consistente para assegurar o restauro da tensão bicipital adequada no período pós-operatório. ${ }^{11}$

Nosso objetivo foi determinar a presença de IG no músculo bíceps braquial após o tratamento das lesões do TCLB (tenodese ou tenotomia) e estabelecer uma relação entre essa possível IG e alterações no comprimento da fibra muscular, presença de deformidades e força.

\section{Materiais e Métodos}

O presente artigo é um estudo observacional e transversal de caso-controle que consiste na análise clínica e de imagem de dois grupos: um composto por pacientes submetidos à tenodese do bíceps no sulco bicipital com parafuso de interferência e outro composto por indivíduos submetidos à tenotomia do bíceps. Os pacientes foram separados de maneira aleatória de acordo com envelopes selados que foram abertos antes de cada cirurgia. Nos dois grupos, os achados foram comparados àqueles do lado contralateral de cada indivíduo (grupo controle).

O presente estudo foi submetido ao Comitê de Ética, sob parecer número CAAE 92179218.8.0000.5505, e todos os pacientes participantes assinaram um termo de consentimento livre e esclarecido.

\section{Escolha de Pacientes}

Os pacientes foram escolhidos com base em uma busca simples nos prontuários médicos da clínica particular do investigador. Os critérios de inclusão foram: concordar e assinar o termo de consentimento livre e esclarecido, ter sido submetido a tenotomia ou tenodese unilateral do bíceps e acompanhamento pós-operatório $>1$ ano.

Os critérios de exclusão foram a presença de lesão do manguito rotador(LMR) ou noTCLB detectada durante o estudo. 
No total, 34 pacientes concordaram em participar do estudo, sendo 17 pacientes no grupo submetido à tenodese e 17 pacientes no grupo submetido à tenotomia. Desses 34 pacientes, 3 foram excluídos do estudo após o exame de ressonância magnética (RM) (2 do grupo de tenotomia e 1 do grupo de tenodese) por apresentarem sinais clínicos e radiológicos de LMR no ombro contralateral, com queixa de dor no braço e perda da força de flexão do cotovelo. Após as exclusões, o grupo de tenodese apresentava 16 pacientes e o grupo de tenotomia, 15 pacientes, totalizando 31 indivíduos analisados ( 31 braços operados e 31 braços contralaterais para controle). Todos os pacientes foram submetidos a cirurgia para tratamento e LMR foi o principal diagnóstico.

\section{Ressonância Magnética}

Todos os exames de RM foram realizados em equipamento Brivo 355 GE de 1,5 Tesla (General Electric, Boston, MA, EUA). Todos os pacientes foram examinados em decúbito dorsal, com os braços posicionados ao longo do corpo e em rotação neutra. Imagens coronais e axiais ponderadas em $\mathrm{T} 1$ foram obtidas de ambos os braços de cada paciente de acordo com um protocolo específico. As sequências coronais ponderadas em T1 foram alinhadas ao corpo da escápula com espaçamento de $3 \mathrm{~mm}$. Essa sequência identificou o ápice da cabeça do úmero e o comprimento do osso para cálculo de sua metade. Uma sequência dupla de cortes axiais ponderados em T1 foi iniciada a partir da borda inferior do acrômio; a primeira sequência foi composta por 25 cortes com espaçamento de $3 \mathrm{~mm}$ e a segunda sequência teve mais 35 cortes com espaçamento de $4 \mathrm{~mm}$. A partir da triangulação com as imagens dos cortes coronais, essa sequência de cortes mais finos ( $3 \mathrm{~mm}$ ) permitiu avaliar a distância entre o ápice da cabeça do úmero e a primeira fibra muscular da porção longa do bíceps na junção musculotendínea (AC-JMT); essa distância foi comparada àquela do lado contralateral, gerando a diferença na distância AC-JMT (-Fig. 1). No grupo de pacientes submetidos a tenodese, a distância entre o ápice da cabeça do úmero e o parafuso de interferência (AC-PI) também foi avaliada.

Como já foi relatado, a RM não permite a individualização do ventre muscular da cabeça longa e da cabeça curta do bíceps e a separação dos demais músculos do compartimento anterior do braço. ${ }^{3,4}$ Por esse motivo, investigamos comparativamente a presença ou não de IG na massa muscular do compartimento anterior do braço usando cortes axiais ponderados em $\mathrm{T} 1$ obtidos o mais próximo possível da metade do comprimento do úmero nos dois grupos. Adaptamos a classificação IG existente ${ }^{12}$ para comparação dos dois braços de cada paciente. Essa classificação sugere 5 graus de IG (grau 0: normal; grau 1: presença de algumas estrias de gordura; grau 2: < 50\% de IG; grau 3: 50\% de IG; grau 4: > 50\% de IG): ${ }^{13}$ embora essa classificação não tenha sido validada com imagens de RM do bíceps, foi usada para esse fim em um estudo recente. ${ }^{4}$ As imagens foram avaliadas por um radiologista experiente, conhecedor do sistema musculoesquelético e com 15 anos de prática clínica.

\section{Avaliação Clínica}

A presença de deformidades nos braços foi classificada como sugerido por Scheibel et al. ${ }^{14}$ como ausente, branda, moderada e grave; para facilitar a análise estatística, subdividimos a presença de deformidades em dois grupos: satisfatória (ausente ou branda) e insatisfatória (moderada ou grave) (-Figs. 2 e 3 ). A força de flexão do cotovelo foi medida com um dinamômetro manual (Fabrication Enterprises, White Plains, New York, USA) (dinamômetro mecânico de mola para aferição de

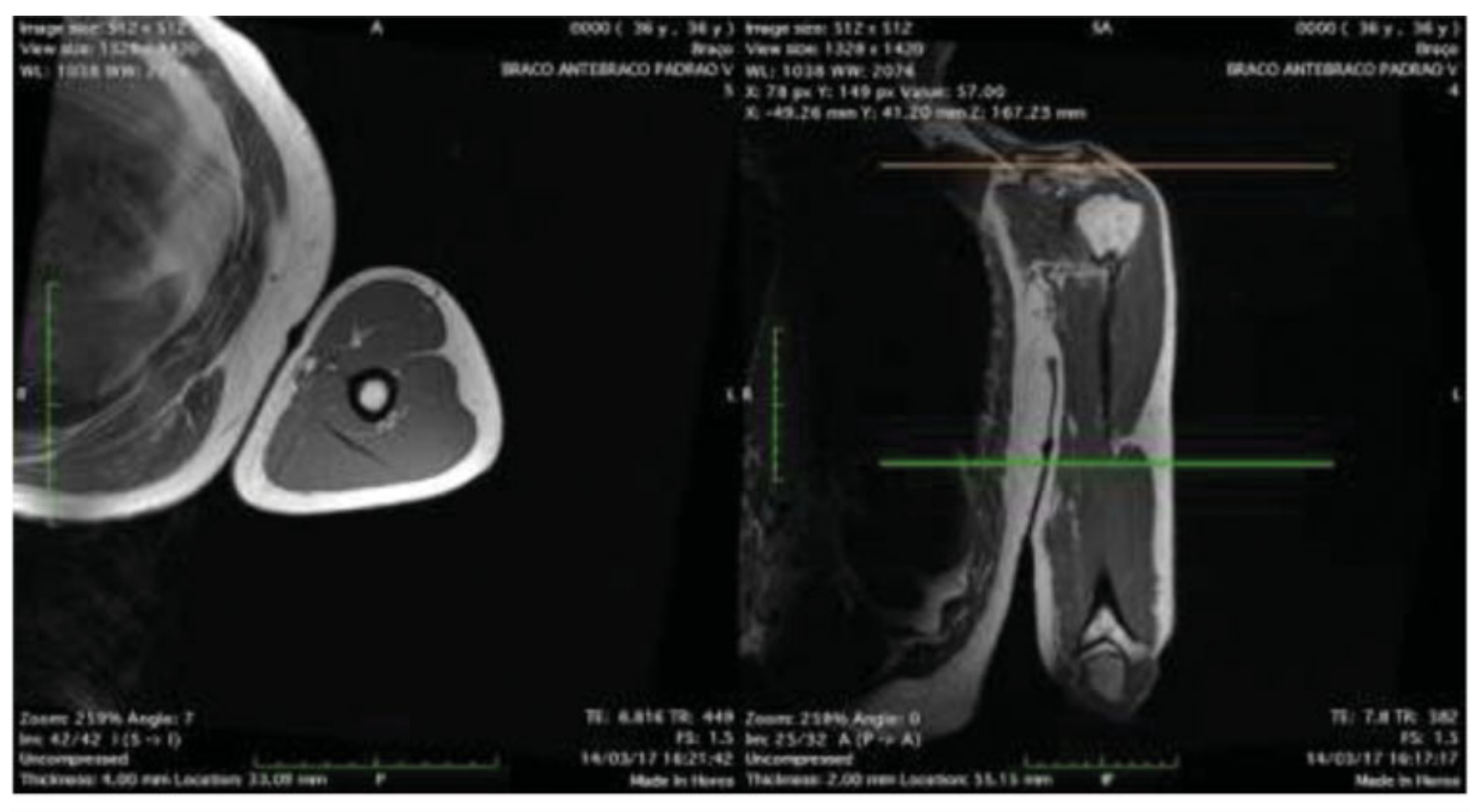

Fonte: Arquivos do autor.

Fig. 1 Distância entre o ápice da cabeça do úmero e a primeira fibra muscular da porção longa do bíceps na junção musculotendínea. 


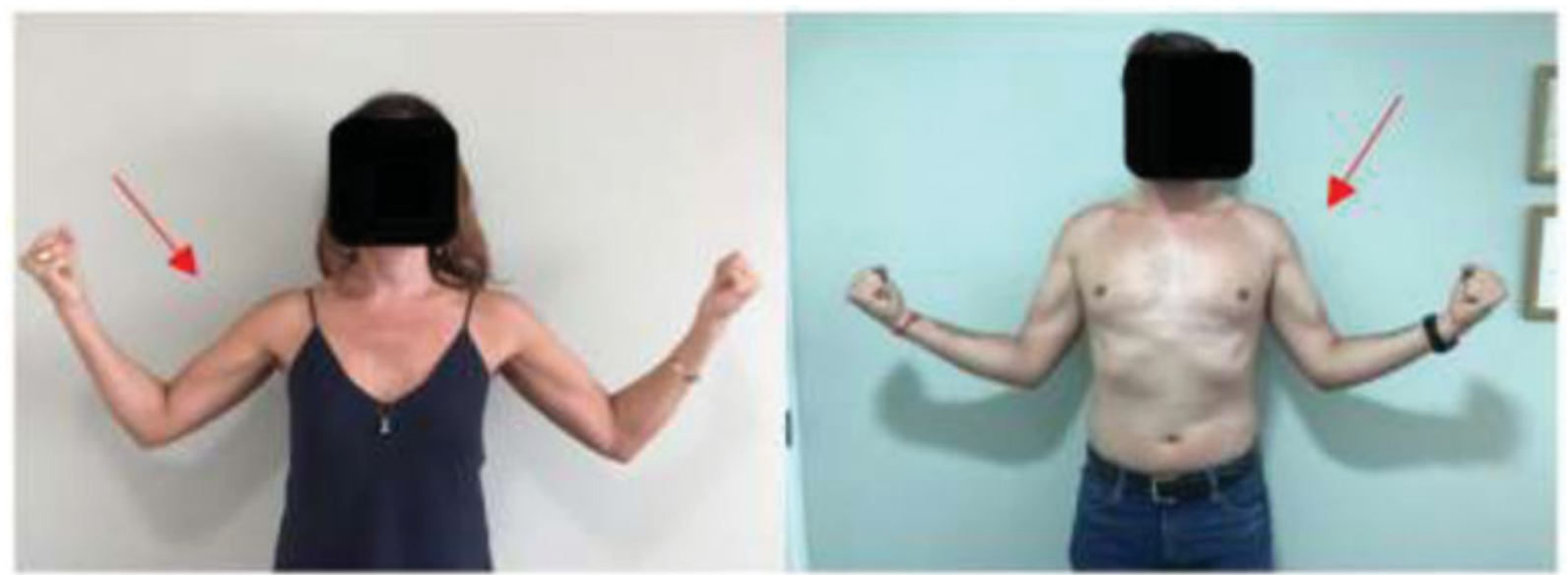

Fonte: Arquivos do autor.

Fig. 2 Deformidade satisfatória.

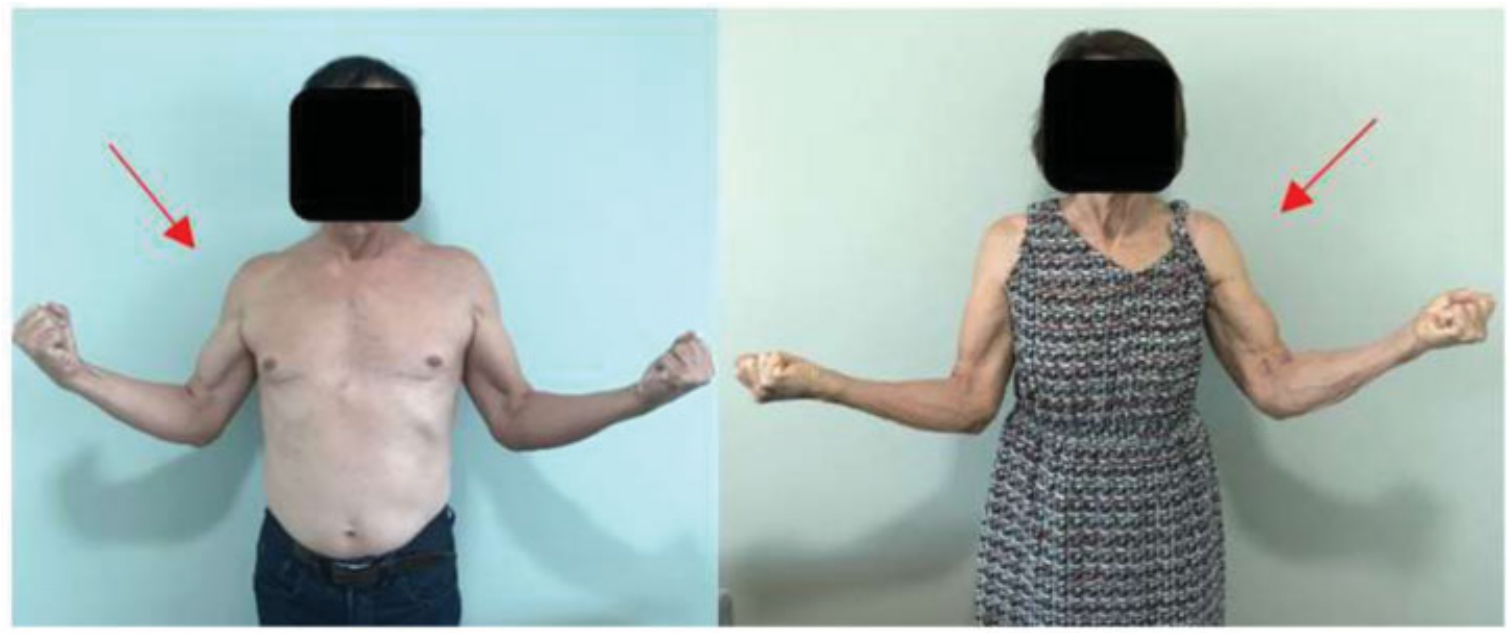

Fonte: Arquivos do autor.

Fig. 3 Deformidade insatisfatória.

pressão/compressão com valor basal de 60 libras) nos dois braços de cada paciente. A dor à palpação do sulco bicipital foi avaliada pelo examinador por compressão digital $5 \mathrm{~cm}$ distal ao canto anterolateral do acrômio com o braço em rotação neutra.

\section{Análise Estatística}

A análise descritiva de todas as variáveis do estudo foi realizada com construção de box plots e cálculo de média, erro padrão, desvio padrão (DP), variância, coeficiente de variação, valor mínimo, $1^{\circ}$ quartil, mediana, $3^{\circ}$ quartil e valor máximo.

Devido ao pequeno tamanho da amostra, o teste exato de Fisher foi usado para determinar a independência entre pares de variáveis. Quando as suposições de uma distribuição normal da média não foram atendidas para as duas amostras ou quando uma das amostras era muito pequena (tamanho igual a 2 ou 3), o teste não paramétrico de Mann-Whitney de igualdade de médias foi empregado nos dois grupos. Se um dos tamanhos da amostra for $<4$, o teste de Mann-Whitney para a diferença de médias pode não ser válido, já que amostras de tamanho muito pequeno não são propícias à rejeição da hipótese testada.
Os respectivos níveis descritivos (valor-p) de todos os testes de hipóteses realizados foram calculados e as hipóteses com níveis descritivos inferiores à significância adotada, de 0,05 , foram rejeitadas. A análise dos dados foi feita no software Minitab v.18 (Minitab, LLC., State College, PA, EUA).

\section{Resultados}

Nos 31 pacientes avaliados, o período pós-operatório médio no momento da realização da RM foi de 5 anos: 5,8 anos (variação de 2 a 11 anos) no grupo submetido a tenotomia e 4,2 anos (variação de 1 a 9 anos) no grupo de tenodese. A idade média dos participantes foi de 60 anos (53 a 77 anos) no grupo de tenotomia e de 50,8 anos (33 a 69 anos) no grupo de tenodese ( $\mathbf{- T a b e l a ~} \mathbf{1}$ ).

\section{Deformidade no Braço}

Entre todos os indivíduos estudados, sete apresentaram deformidades insatisfatórias (moderadas ou graves) no braço operado. A diferença na distância AC-JMT variou de $-0,6 \mathrm{~cm}$ a $3,1 \mathrm{~cm}$ (média de 0,34 cm). Não observamos relação significativa entre a presença de deformidades satisfatórias e a diferença na 
Tabela 1 Características da amostra

\begin{tabular}{|l|l|l|l|l|l|l|l|}
\hline Grupo & $\begin{array}{l}\text { Número de } \\
\text { pacientes }\end{array}$ & $\begin{array}{l}\text { Período pós- } \\
\text { operatório médio }\end{array}$ & $\begin{array}{l}\text { Indicação } \\
\text { para cirurgia }\end{array}$ & $\begin{array}{l}\text { Idade média } \\
\text { à cirurgia }\end{array}$ & $\begin{array}{l}\text { Distância } \\
\text { Média }\end{array}$ & Homens & Mulheres \\
\hline Tenotomia & 15 & 5,8 anos (2-5 anos) & $\begin{array}{l}\text { Lesão do manguito } \\
\text { rotador }\end{array}$ & $\begin{array}{l}63 \text { anos } \\
(53-77 \text { anos) }\end{array}$ & 5 & $3(20 \%)$ & $12(80 \%)$ \\
\hline Tenodese & 16 & 4,2 anos (1-9 anos) & $\begin{array}{l}\text { Lesão do manguito } \\
\text { rotador }\end{array}$ & $\begin{array}{l}50,8 \text { anos } \\
(33-69 \text { anos) }\end{array}$ & 1 & $15(93,5 \%)$ & $1(6,5 \%)$ \\
\hline
\end{tabular}

Fonte: Preparada pelo autor.

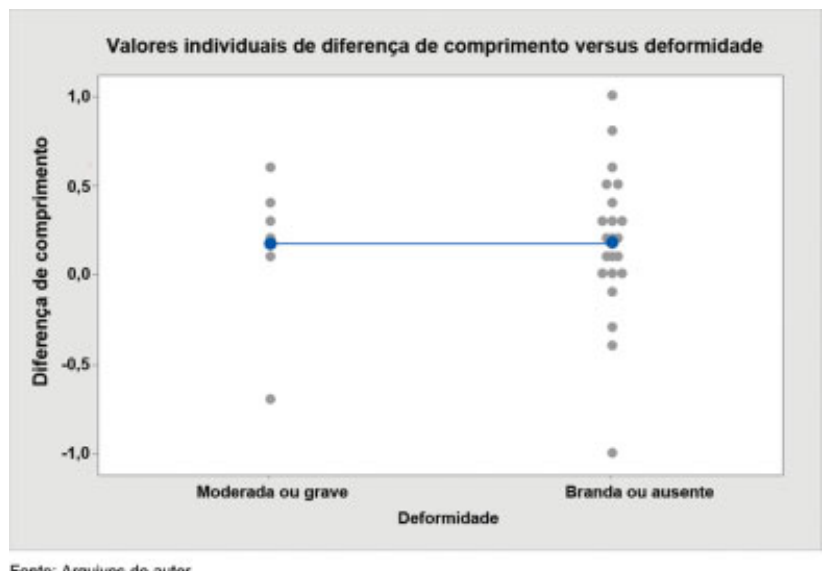

Fonte: Arquivos do autor.

Fig. 4 Valores individuais de diferença de comprimento versus deformidade.

distância AC-JMT ( $p=0,124)$ ou entre a incidência de deformidades insatisfatórias e a diferença na distância AC-JMT $(p=0,077)$. As médias das diferenças na distância AC-JMT foram semelhantes nos pacientes que apresentaram deformidades satisfatórias $(0,175 \mathrm{~cm})$ e insatisfatórias $(0,171 \mathrm{~cm})(p=0,984)$. Ambas apresentaram distribuição normal (-Fig. 4).

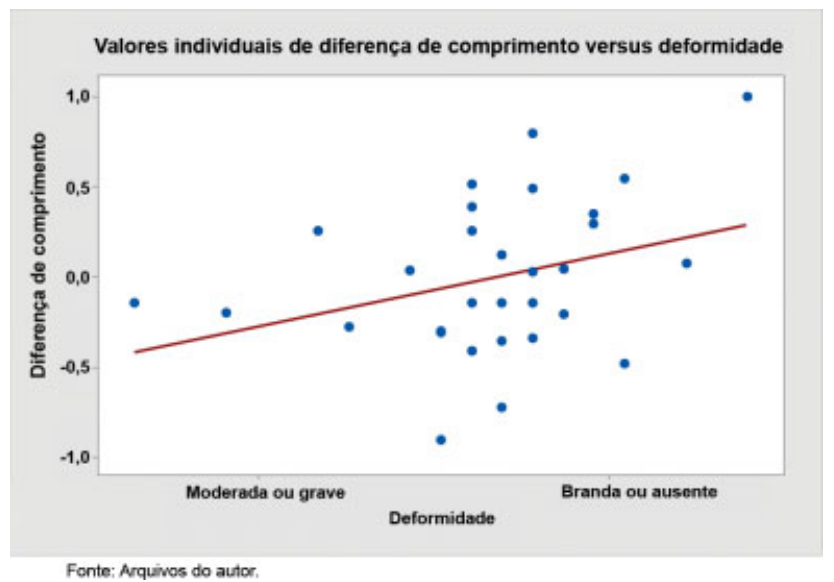

Fig. 6 Porcentagem de força do braço operado versus diferença de comprimento.

\section{Infiltração Gordurosa}

Nenhum paciente em nenhum dos grupos apresentou alterações na massa muscular do compartimento anterior do braço e não houve evidências de IG em imagens axiais ponderadas em T1 à altura de metade do comprimento total do braço. Todos os casos foram classificados como grau 0 (-Fig. 5).

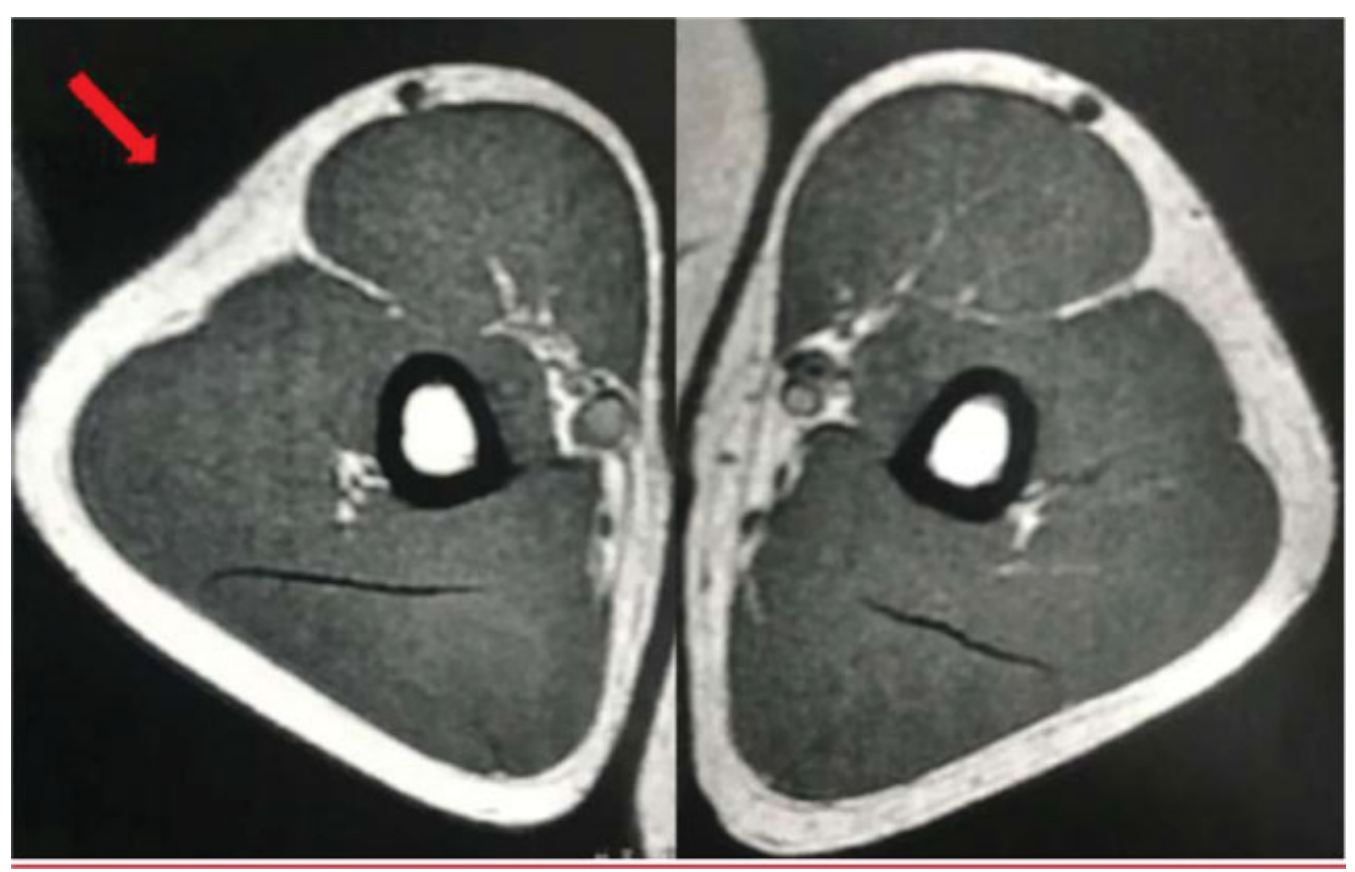

Fig. 5 Infiltração gordurosa. 


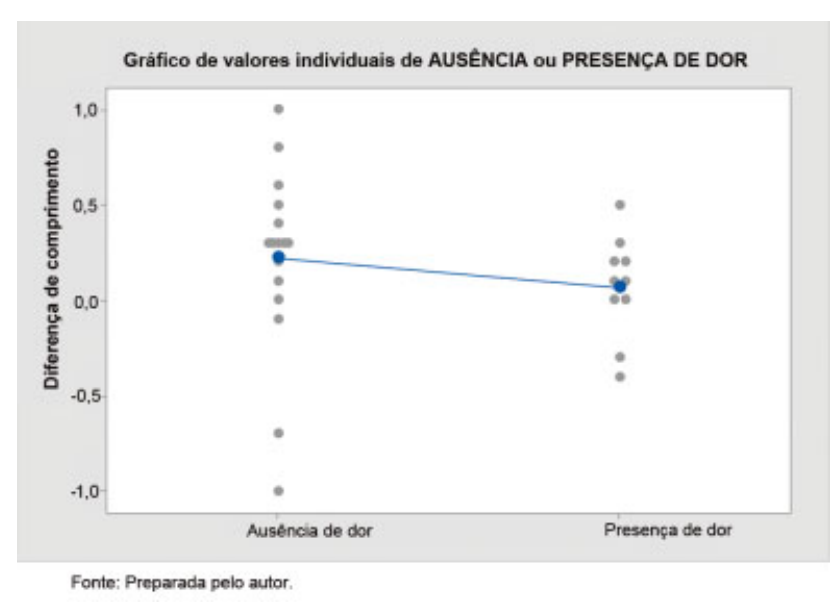

Fig. 7 Gráfico de valores individuais de ausência ou presença de dor.

\section{Força de Flexão}

O percentual de força de flexão dos braços operados não apresentou relação linear com a diferença na distância ACJMT $(p=0,070)$ (-Fig. 6). Não houve diferença na força percentual média entre os grupos $(p=0,399)$ : 106,7\% (variação de 92,86 a $137,57 \%$ ) no grupo submetido a tenodese e 102,4\% (variação de 75 a 130,77\%) no grupo de tenotomia. A distribuição foi normal em ambos os grupos.

\section{Dor à Palpação do Sulco Intertubercular}

Não observamos relação significativa entre dor à palpação do sulco bicipital e a diferença na distância AC-JMT $(p=0,103)$ (-Fig. 7). Não houve correlação significativa entre a incidência de dor no sulco bicipital e a força percentual média de flexão do cotovelo do braço operado ( $p=0,062)$.

\section{Discussão}

Não encontramos evidências de IG na massa muscular do compartimento anterior dos braços submetidos a procedimentos para o tratamento das lesões do TCLB em um período médio de acompanhamento pós-operatório de 5 anos. Não houve diferença na aparência do músculo na RM após tenotomia ou tenodese do bíceps. Assim, as variações no comprimento da massa muscular do bíceps detectadas pelo presente estudo (diferença na distância AC-JMT) não contribuíram para o aparecimento de IG no ventre do músculo. Além disso, não foi possível estabelecer uma correlação entre a discrepância no comprimento do bíceps medido à RM e dor residual, perda de força de flexão ou deformidade do braço.

Nossas imagens de RM foram obtidas de ambos os braços e de todos os 31 pacientes; a massa muscular do lado operado foi comparada à massa muscular contralateral, sem alterações significativas no bíceps e no manguito rotador. Investigamos quantitativamente a retração do ventre muscular e sua possível relação com a presença de IG.

Sete pacientes apresentavam deformidades insatisfatórias (moderadas ou graves) no braço operado. As médias das diferenças de distância AC-JMT foram semelhantes nos indivíduos com deformidades satisfatórias e insatisfatórias. Com isso, conclui-se que a distância AC-JMT não é o único aspecto a determinar a satisfação do paciente.

No presente estudo, realizamos exames de RM em ambos os braços dos 31 pacientes para comparação da massa muscular do compartimento anterior dos dois membros e da variação no comprimento da massa muscular do bíceps com base na diferença de distância AC-JMT. Não houve relação entre a diferença na distância AC-JMT e IG; da mesma maneira, não houve relação entre IG e a presença de deformidade do braço. Igualmente, nos 31 pacientes avaliados, não houve sinais de IG após um período pós-operatório médio de 5 anos. Esse achado sugere que lesões crônicas do bíceps podem ser reparadas, mantendo boa potência e função muscular, pois a musculatura é preservada ao longo dos anos mesmo em caso de retração. Também não observamos correlação entre IG e deformidade do braço.

A ausência crônica de carga e a tenotomia simples dos músculos do manguito rotador podem levar ao desenvolvimento de IG e a uma redução significativa do volume muscular. ${ }^{2,15}$ A cabeça longa do bíceps se origina no tubérculo supraglenoide proximal, se funde à cabeça curta e se insere distalmente na tuberosidade do rádio, cruzando duas articulações; portanto, mesmo em caso de soltura de sua extremidade proximal, a extremidade distal permanece inserida, gerando carga e ativando as fibras musculares.

A tenotomia pode ser acompanhada por dor residual porque parte da força gerada pela contração muscular não é transmitida a um tendão ligado a osso $;^{3}$ há relato de aumento na incidência de câimbras no bíceps. ${ }^{16}$ Na tenodese, a dor pode ser causada pela persistência da sinovite no sulco bicipital, principalmente se a fixação for realizada na margem articular ou na porção mais proximal do sulco intertubercular. ${ }^{9}$ Independentemente da técnica, em caso de retração excessiva do ventre muscular, uma eventual tensão crônica no ramo comum do nervo musculocutâneo pode causar dor. Não observamos relação significativa entre dor à palpação do sulco intertubercular e a diferença na distância AC-JMT $(p=0,103)$. A dor residual relacionada ao bíceps pode ser encontrada em 19\% dos casos de tenotomia e em $24 \%$ dos casos de tenodese. ${ }^{9}$ Em nosso estudo, a dor à palpação do sulco intertubercular foi detectada em 10 dos 31 pacientes, 3 pertencentes ao grupo de tenodese $(18,75 \%)$ e 7 ao grupo de tenotomia (46,6\%); no entanto, essa diferença não foi significativa $(p=0,135)$, talvez devido ao tamanho da amostra.

Nosso estudo comparou o percentual de força de flexão do cotovelo à diferença na distância AC-JMT, sem estabelecimento de uma relação significativa $(p=0,070)$. Ainda há controvérsias acerca da existência de uma diferença clínica na perda de força após a tenotomia ou tenodese. ${ }^{9}$ Shank et al. ${ }^{17}$ não encontraram diferença de força na comparação após a realização de tenodese ou tenotomia. Outro estudo detectou uma perda de $20 \%$ da força de flexão do cotovelo após a tenotomia ${ }^{16}$ Embora não seja o objetivo do nosso estudo, comparamos individualmente os resultados dos dois grupos e não encontramos diferença significativa no percentual de força de flexão do cotovelo $(p=0,311)$. O grupo 
submetido a tenotomia apresentou força média de 102,4\% em comparação ao lado contralateral, enquanto o grupo de tenodese teve uma força média um pouco superior, de $106,7 \%$.

Nosso estudo tem algumas limitações. A princípio, considerando que a JMT pode se estender por $\sim 8 \mathrm{~cm}^{11}$ estipulamos como medida padrão as primeiras fibras musculares do bíceps identificadas no corte axial ponderado em $\mathrm{T} 1$; no entanto, esta medida pode sofrer variações anatômicas individuais. Para minimizar esses efeitos, todos os exames de RM foram realizados no mesmo equipamento, seguindo o mesmo protocolo, e foram avaliados pelo mesmo radiologista. Uma segunda limitação foi a ausência de avaliação histológica das fibras musculares. Embora a avaliação histológica talvez seja o padrão-ouro para avaliação de IG, sua execução seria eticamente impossível; além disso, a análise por RM tem sido bastante utilizada na investigação do trofismo muscular e está bem estabelecida na cirurgia de ombro e de cotovelo. Uma terceira limitação é o uso do dinamômetro manual, que não é a ferramenta perfeita, mas dá uma boa ideia da força de flexão do cotovelo. $O$ número de indivíduos pode ter sido insuficiente para algumas análises secundárias; no entanto, foi absolutamente suficiente para o objetivo inicial e principal de avaliação da IG do músculo bíceps braquial.

\section{Conclusão}

Em um período médio de acompanhamento de 5 anos, nenhum dos pacientes avaliados apresentou evidência de IG na massa muscular do compartimento anterior do braço após tenodese ou tenotomia do bíceps.

Não houve diferença entre tenotomia e tenodese em relação ao padrão de massa muscular observado na RM.

Não foi possível estabelecer uma correlação entre a discrepância do comprimento do músculo bíceps medido na $\mathrm{RM}$, a presença de deformidades, a força e a presença de IG no compartimento anterior do braço.

\section{Suporte Financeiro}

Não houve suporte financeiro de fontes públicas, comerciais, ou sem fins lucrativos.

Conflito de Interesses

Os autores declaram não haver conflito de interesses.

\section{Referências}

1 Lim TK, Moon ES, Koh KH, Yoo JC. Patient-related factors and complications after arthroscopic tenotomy of the long head of the biceps tendon. Am J Sports Med 2011;39(04):783-789

2 Gerber C, Meyer DC, Flück M, et al. Muscle degeneration associated with rotator cuff tendon release and/or denervation in sheep. Am J Sports Med 2017;45(03):651-658

3 Deutch SR, Gelineck J, Johannsen HV, Sneppen O. Permanent disabilities in the displaced muscle from rupture of the long head tendon of the biceps. Scand J Med Sci Sports 2005;15(03):159-162

4 The B, Brutty M, Wang A, et al. Biceps muscle fatty infiltration and atrophy. A midterm review after arthroscopic tenotomy of the long head of the biceps. Arthroscopy 2015;31(03):477-481

5 Sanders B, Lavery KP, Pennington S, Warner JJ. Clinical success of biceps tenodesis with and without release of the transverse humeral ligament. J Shoulder Elbow Surg 2012;21(01):66-71

6 Hsu AR, Ghodadra NS, Provencher MT, Lewis PB, Bach BR. Biceps tenotomy versus tenodesis: a review of clinical outcomes and biomechanical results. J Shoulder Elbow Surg 2011;20(02):326-332

7 Neer CS II. Anterior acromioplasty for the chronic impingement syndrome in the shoulder: a preliminary report. J Bone Joint Surg Am 1972;54(01):41-50

8 Elser F, Braun S, Dewing CB, Giphart JE, Millett PJ. Anatomy, function, injuries, and treatment of the long head of the biceps brachii tendon. Arthroscopy 2011;27(04):581-592

9 Slenker NR, Lawson K, Ciccotti MG, Dodson CC, Cohen SB. Biceps tenotomy versus tenodesis: clinical outcomes. Arthroscopy 2012; 28(04):576-582

10 Werner BC. Editorial commentary: how can I tenodese the biceps tendon of the shoulder? Let me count the ways. Arthroscopy 2018;34(06):1762-1763

11 Lafrance R, Madsen W, Yaseen Z, Giordano B, Maloney M, Voloshin I. Relevant anatomic landmarks and measurements for biceps tenodesis. Am J Sports Med 2013;41(06):1395-1399

12 Fuchs B, Weishaupt D, Zanetti M, Hodler J, Gerber C. Fatty degeneration of the muscles of the rotator cuff: assessment by computed tomography versus magnetic resonance imaging. J Shoulder Elbow Surg 1999;8(06):599-605

13 Kang JR, Gupta R. Mechanisms of fatty degeneration in massive rotator cuff tears. J Shoulder Elbow Surg 2012;21(02):175-180

14 Scheibel M, Schröder RJ, Chen J, Bartsch M. Arthroscopic soft tissue tenodesis versus bony fixation anchor tenodesis of the long head of the biceps tendon. Am J Sports Med 2011;39(05):1046-1052

15 Meyer DC, Hoppeler H, von Rechenberg B, Gerber C. A pathomechanical concept explains muscle loss and fatty muscular changes following surgical tendon release. J Orthop Res 2004;22(05): 1004-1007

$16 \mathrm{Koh} \mathrm{KH}, \mathrm{Ahn} \mathrm{JH}, \mathrm{Kim}$ SM, Yoo JC. Treatment of biceps tendon lesions in the setting of rotator cuff tears: prospective cohort study of tenotomy versus tenodesis. Am J Sports Med 2010;38 (08):1584-1590

17 Shank JR, Singleton SB, Braun S, et al. A comparison of forearm supination and elbow flexion strength in patients with long head of the biceps tenotomy or tenodesis. Arthroscopy 2011;27(01):9-16 